\title{
Enhancing inactivation rather than reducing activation of Nav1.7 channels by a clinically effective analgesic CNV1014802
}

\author{
Yue-ming ZHENG ${ }^{1}$, Wan-fu WANG ${ }^{1,2}$, Yan-fen $\mathrm{LI}^{1,3}$, Yong $\mathrm{YU}^{4, *}$, Zhao-bing GAO ${ }^{1, \text { * }}$ \\ ${ }^{1}$ CAS Key Laboratory of Receptor Research, State Key Laboratory of Drug Research, Shanghai Institute of Materia Medica, Chinese \\ Academy of Sciences, Shanghai 201203, China; ${ }^{2}$ Shanghai Key Laboratory of New Drug Design, School of Pharmacy, East China \\ University of Science and Technology, Shanghai 200237, China; ${ }^{3}$ Shanghai Key Laboratory of Bio-Energy Crops, School of Life \\ Sciences, Shanghai University, Shanghai 200444, China; ${ }^{4}$ Department of Neurosurgery, Zhongshan Hospital, Fudan University, \\ Shanghai 200032, China
}

\begin{abstract}
The Nav1.7 channel represents a promising target for pain relief. In the recent decades, a number of Nav1.7 channel inhibitors have been developed. According to the effects on channel kinetics, these inhibitors could be divided into two major classes: reducing activation or enhancing inactivation. To date, however, only several inhibitors have moved forward into phase 2 clinical trials and most of them display a less than ideal analgesic efficacy, thus intensifying the controversy regarding if an ideal candidate should preferentially affect the activation or inactivation state. In the present study, we investigated the action mechanisms of a recently clinically confirmed inhibitor CNV1014802 using both electrophysiology and site-directed mutagenesis. We found that CNV1014802 inhibited Nav1.7 channels through stabilizing a nonconductive inactivated state. When the cells expressing Nav1.7 channels were hold at $70 \mathrm{mV}$ or $120 \mathrm{mV}$, the half maximal inhibitory concentration $\left(\mathrm{IC}_{50}\right.$ ) values (with 95\% confidence limits) were 1.77 (1.20-2.33) and 71.66 (46.85-96.48) $\mathrm{mmol} / \mathrm{L}$, respectively. This drug caused dramatic hyperpolarizing shift of channel inactivation but did not affect activation. Moreover, CNV1014802 accelerated the onset of inactivation and delayed the recovery from inactivation. Notably, application of CNV1014802 (30 $\mu \mathrm{mol} / \mathrm{L})$ could rescue the Nav1.7 mutations expressed in $\mathrm{CHO}$ cells that cause paroxysmal extreme pain disorder (PEPD), thereby restoring the impaired inactivation to those of the wild-type channel. Our study demonstrates that CNV1014802 enhances the inactivation but does not reduce the activation of Nav1.7 channels, suggesting that identifying inhibitors that preferentially affect inactivation is a promising approach for developing drugs targeting Nav1.7.
\end{abstract}

Keywords: Nav1.7 channels; analgesics; CNV1014802; inactivated state; paroxysmal extreme pain disorder

Acta Pharmacologica Sinica (2018) 39: 587-596; doi: 10.1038/aps.2017.151; published online 2 Nov 2017

\section{Introduction}

Voltage-gated sodium (Nav) channels play a fundamental role in the generation and propagation of electrical impulses in excitable cells $^{[1]}$. The Nav1.7 channel, one of the nine Nav isoforms (Nav1.1-Nav1.9), encoded by SCN9A, is preferentially expressed in nociceptive dorsal root ganglion neurons and sympathetic ganglion neurons ${ }^{[2-4]}$. A number of human genetic mutations in Nav1.7 have been linked to either hyposensitive or hypersensitive pain states ${ }^{[4]}$. Gain-of-function mutations have been found to cause inherited erythromelalgia (IEM) ${ }^{[5]}$,

\footnotetext{
*To whom correspondence should be addressed.

E-mail zbgao@simm.ac.cn (Zhao-bing GAO);

yu.yong@zs-hospital.sh.cn (Yong YU)

Received 2017-07-19 Accepted 2017-09-03
}

paroxysmal extreme pain disorder (PEPD) ${ }^{[6]}$ and small-fiber neuropathy $(\mathrm{SFN})^{[7]}$. In contrast, loss-of-function recessive mutations cause congenital insensitivity to pain (CIP), in which individuals do not experience any form of pain ${ }^{[8,9]}$. These genetic observations in conjunction with findings from other functional studies suggest that the Nav1.7 channel may be a promising target, and intense efforts have been undertaken to develop inhibitors against Nav1.7 to treat pain ${ }^{[10-12]}$.

As Nav1.7, a threshold channel in nociceptive neurons, plays a crucial role in pain generation, inhibitors that reduce activation and/or enhance inactivation of the Nav1.7 channel are thought to be effective at dampening neuron excitability and attenuating pain ${ }^{[4,13]}$. In recent decades, a few Nav1.7 inhibitors, such as antibodies, toxins and small molecules, that preferentially affect channel activation or inactivation have 
been developed ${ }^{[10,12,14]}$. However, their analgesic effects are disappointing ${ }^{[11,15,16]}$. For example, ProTx-II, a selective tarantula venom peptide, strongly right-shifts channel activation ${ }^{[17]}$. However, it does not exhibit analgesia after intravenous or intrathecal administration in a Complete Freund's Adjuvantinduced inflammatory pain model despite the fact that it potently blocks Nav1.7 currents and relives OD-1-induced pain after local administration ${ }^{[17]}$. Remarkably, PF-05089771, a potent and selective Nav1.7 inhibitor developed by Pfizer, is suggested to inhibit Nav1.7 currents by hindering channel recovery from inactivation similar to other aryl sulfonamide inhibitors ${ }^{[18,19]}$. Even though it relieves pain syndromes in a small group of patients with IEM, Pfizer has discontinued its clinical study due to the lack of efficacy for diabetic neuropathy pain ${ }^{[12,20]}$. These and other disappointing data not only highlight the difficulty in the discovery of analgesics that target the Nav1.7 channel but also intensify the controversy on if an ideal candidate preferentially affecting the activation or inactivation state will relieve pain ${ }^{[21]}$.

CNV1014802 is a state-dependent inhibitor of Nav1.7 channels developed by Convergence Pharmaceuticals ${ }^{[22]}$. Clinical study in a phase 2 trial of patients with painful lumbosacral radiculopathy (LSR) demonstrated that CNV1014802 is well tolerated and produces a remarkable reduction in pain compared to placebo ${ }^{[23]}$. However, whether the inhibitory effect of CNV1014802 on Nav1.7 channels is through modification of channel kinetics remains unclear. In the present study, we set out to characterize the electrophysiological properties of CNV1014802 and to probe the relationship between channel kinetics and its pharmacological application.

\section{Material and methods Materials}

CNV1014802 was synthesized in the laboratory of Prof Jian LI (East China University of Science and Technology, Shanghai, China). The purity of the synthetic CNV1014802 was $\geq 98 \%$, which was assessed by HPLC. The compound was dissolved and stored in dimethyl sulfoxide (DMSO) to produce 100 $\mathrm{mmol} / \mathrm{L}$ stock solutions that were then diluted into the bath solution to obtain the final concentrations. The final concentration of DMSO $(\leq 0.3 \%)$ was tested and verified to have no effect on sodium currents. All other chemicals were obtained from Sigma-Aldrich (St Louis, MO, USA).

\section{cDNA and mutagenesis}

The plasmid carrying the human Nav1.7 cDNA insert was a gift from Dr Norbert KLUGBAUER (Albert-Ludwigs-Universität Freiburg, Freiburg, Germany) ${ }^{[2]}$. Point mutations in the Nav1.7 channel were individually constructed by recombinant PCR and confirmed by sequencing.

\section{Cell culture and transfection}

Human embryonic kidney 293 (HEK293) cells stably expressing hNav1.7 were a gift from Dr Hai-bo YU (Institute of Materia Medica, Beijing, China). Cells were grown in high-glucose DMEM (Gibco, Carlsbad, CA, USA) supplemented with 10\% fetal bovine serum and were selected with $300 \mu \mathrm{g} / \mathrm{mL}$ of the antibiotic Hygromycin B (Invitrogen, Carlsbad, CA, USA) under standard tissue culture conditions $\left(5 \% \mathrm{CO}_{2}, 37^{\circ} \mathrm{C}\right)$. For the functional expression of Nav1.7 mutants, Chinese hamster ovary (CHO) cells were used and cultured in 50/50 DMEM/ F-12 (Gibco, Carlsbad, CA, USA) supplemented with 10\% fetal bovine serum. Two days prior to recording, the constructs were transfected into $\mathrm{CHO}$ cells with Lipofectamine reagent (Invitrogen, Carlsbad, CA, USA), according to the manufacture's protocol. A GFP construct was co-transfected to aid in the identification of transfected cells by fluorescence microscopy. Cells were seeded onto poly-L-lysine-coated glass coverslips before they were used for electrophysiology recording.

\section{Electrophysiology}

Whole-cell patch-clamp recordings were conducted at room temperature using an Axopatch 200B patch clamp amplifier (Molecular Devices, Sunnyvale, CA, USA). Pipettes were pulled from borosilicate glass capillaries (World Precision Instruments, Sarasota, FL, USA) with an electrode resistance typically ranging from 1.5 to $4 \mathrm{M} \Omega$. The recording pipette intracellular solution contained the following (in $\mathrm{mmol} / \mathrm{L}$ ): $140 \mathrm{CsF}, 10 \mathrm{NaCl}, 10$ HEPES, 1.1 EGTA and 20 glucose ( $\mathrm{pH}$ 7.3 adjusted by $\mathrm{CsOH}$ ); the bath or extracellular solution contained the following (in mmol/L): $140 \mathrm{NaCl}, 3 \mathrm{KCl}, 1 \mathrm{MgCl}_{2}, 1$ $\mathrm{CaCl}_{2}, 10$ HEPES and 20 glucose (pH 7.3 adjusted by $\mathrm{NaOH}$ ). During the recording, the bath solution was continuously perfused using a BPS perfusion system (ALA Scientific Instruments, Westburg, NY, USA). Recording was performed after a 5-min equilibration period at $-80 \mathrm{mV}$ after breaking into the whole-cell configuration. Currents were acquired at a $50-\mathrm{kHz}$ sampling frequency and filtered at $2 \mathrm{kHz}$. Series resistance compensation was used and set to $80 \%$. P/N subtraction was never applied throughout the experiment. An unsaturated $\mathrm{IC}_{90}$ concentration $(10 \mu \mathrm{mol} / \mathrm{L})$ was applied throughout the whole study unless otherwise stated. If a change in activation was not observed at $10 \mu \mathrm{mol} / \mathrm{L}$, a higher concentration of the drug $(30 \mu \mathrm{mol} / \mathrm{L})$ was further administered to confirm the lack of an effect. This higher concentration was also used in the parallel experiments, such as the inactivation recordings.

\section{Data and statistical analysis}

The data and statistical analysis complied with the recommendations on experimental design and analysis in pharmacology ${ }^{[24]}$. Patch-clamp data were processed using Clampfit 10.3 (Molecular Device, Sunnyvale, CA, USA) and analyzed with GraphPad Prism 5.0 (GraphPad Software, San Diego, CA, USA). Peak inward currents obtained from activation protocols were converted to conductance values using the equation: $G=I /\left(V-V_{\mathrm{Na}}\right) . \quad V_{\mathrm{Na}}$ indicates the reversal potential of sodium currents. To measure inactivation, the peak currents after different pre-pulses were normalized and plotted as a function of voltage. To quantify these biophysical properties, we fitted the activation and steady-state inactivation curves with the Boltzmann equation: $G=G_{\min }+\left(G_{\max }-G_{\min }\right) /\left(1+\exp \left[\left(V_{\mathrm{m}}-\right.\right.\right.$ $\left.\left.V_{1 / 2}\right) / k\right]$ ), where $G_{\max }$ is the maximum conductance, $G_{\min }$ is the 
minimum conductance, $V_{1 / 2}$ is the voltage for reaching $50 \%$ of the maximum conductance, and $k$ is the slope factor. Doseresponse curves were fitted with a 3-parameter Hill equation: $\mathrm{Y}=$ Bottom $+($ Top-Bottom $) /\left(1+10^{\wedge}\left(\mathrm{X}-\log \mathrm{IC}_{50}\right)\right)$, where Bottom and Top are the minimum and maximum inhibition, respectively, $\mathrm{X}$ is the $\log$ of concentration, $\mathrm{Y}$ is the value of $I_{\mathrm{drug}} / I_{\text {control}}$, and $\mathrm{IC}_{50}$ is the drug concentration producing a half-maximum response. The onset of inactivation in the absence and presence of CNV1014802 was well described by a one-phase and two-phase exponential decay, respectively. Recovery from inactivation with and without CNV1014802 was well fitted by a two-phase exponential decay. The results are presented as the mean $\pm S E M$. The significance was estimated using 2-tailed $t$ tests (paired or unpaired), and a $P$-value $<0.05$ was considered statistically significant. Detailed information is provided in the figure legends.

\section{Results}

CNV1014802 exerts a state-dependent inhibition on Nav1.7 channels

The inhibitory activity of CNV1014802 on Nav1.7 channels was examined using the whole-cell voltage-clamp technique. To elicit Nav1.7 currents, a $20-\mathrm{ms}$ test pulse to $0 \mathrm{mV}$ from holding potentials ranging from $-80 \mathrm{mV}$ to $-120 \mathrm{mV}$ was applied. At a holding potential of $-80 \mathrm{mV}$, which would inactivate approximately $20 \%$ of Nav1.7 channels, the peak currents dramatically decreased after bath perfusion of $30 \mu \mathrm{mol} / \mathrm{L}$ CNV1014802 (Figure 1A). However, when the holding potential was clamped at $-120 \mathrm{mV}$, a membrane potential that would prevent channel inactivation, the block was less dramatic (Figure 1A). The reductions in the peak currents caused by drug application were $58 \% \pm 6 \%$ and $26 \% \pm 2 \%$ when tested from the holding potentials of $-80 \mathrm{mV}$ and $-120 \mathrm{mV}$, respectively (Figure 1B). The inhibitory effects in both conditions were completely washed out when the holding potential was changed to -120 $\mathrm{mV}$. The differential inhibitory potencies for the resting and inactivated channels suggest that CNV1014802 inhibits Nav1.7 channels in a state-dependent manner.

\section{CNV1014802 does not affect steady-state activation}

To understand if CNV1014802 modifies the kinetics of Nav1.7 channels, its effects on steady-state activation were first investigated. Activation currents were elicited by step stimulations ranging from $-80 \mathrm{mV}$ to $+15 \mathrm{mV}$ from a holding potential of $-120 \mathrm{mV}$ in 5-mV increments for $50 \mathrm{~ms}$. Representative current traces recorded before and after bath perfusion of $30 \mu \mathrm{mol} / \mathrm{L}$ CNV1014802 are shown in Figure 2A. Normalized currentvoltage $(I-V)$ curves showed that CNV1014802 caused a $27 \% \pm 3 \%$ decrease in the peak current but had little or no effect on the voltage dependence of activation (Figure 2B). Activation curves were fitted with the Boltzmann equation and demonstrated no significant changes in the half-activation voltage after the application of CNV1014802 (Figure 2C). The $V_{1 / 2}$ values before and during perfusion of $30 \mu \mathrm{mol} / \mathrm{L}$ CNV1014802 were $-21.64 \pm 0.96 \mathrm{mV}$ and $-23.26 \pm 0.85 \mathrm{mV}$, respectively. These results demonstrate that CNV1014802 does not affect the acti-
A
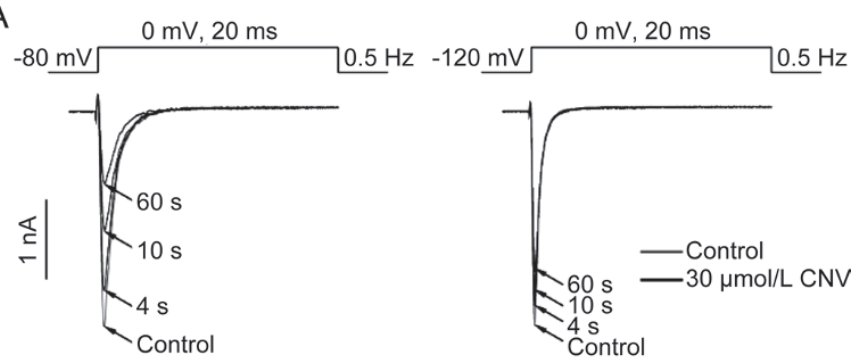

B

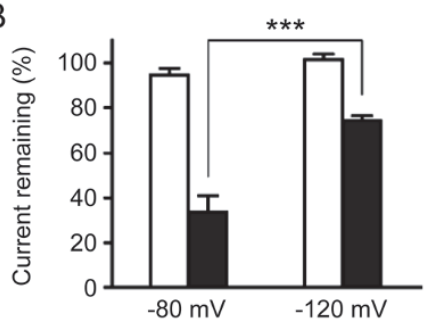

C

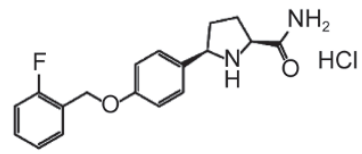

CNV1014802 (CNV)

Figure 1. CNV1014802 inhibition of Nav1.7 channels. (A) Representative Nav1.7 currents before and after bath perfusion of 30 $\mu \mathrm{mol} / \mathrm{L}$ CNV1014802 from the indicated holding potentials. (Inset) The experimental protocol. The currents were elicited by a $20 \mathrm{~ms}$ test pulse to $0 \mathrm{mV}$ from a holding potential of $-80 \mathrm{mV}$ or $-120 \mathrm{mV}$. (B) Bar graph showing inhibition of $30 \mu \mathrm{mol} / \mathrm{L}$ CNV1014802 (black) on Nav1.7 channels at indicated holding potentials compared with time-dependent controls (white). CNV1014802 was applied for 30-60 s to reach steady state block. ( $n=8$ for each group, ${ }^{* * *} P<0.0001$, unpaired 2 -tailed t test). (C) Chemical structure of CNV1014802.

A

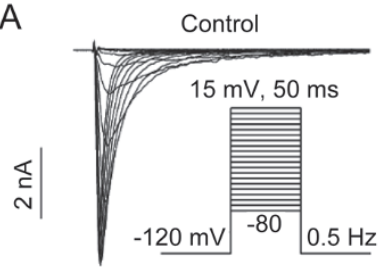

$\mathrm{B}$

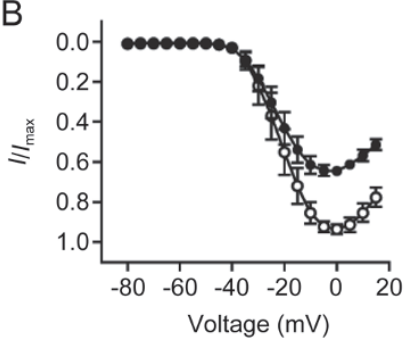

$30 \mu \mathrm{mol} / \mathrm{L}$ CNV
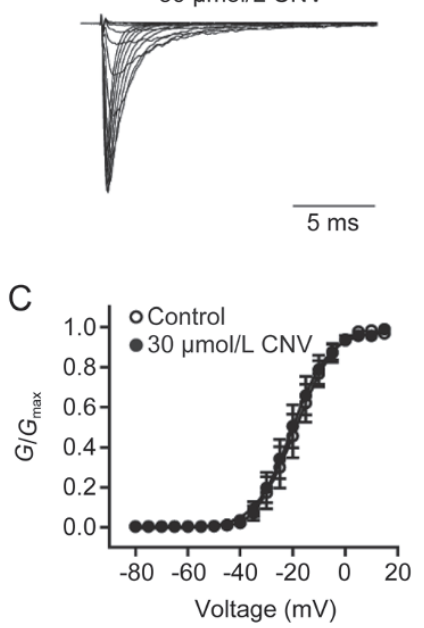

Figure 2. Effects of CNV1014802 on Nav1.7 channel activation. (A) Representative activation current traces of Nav1.7 in the absence or presence of $30 \mu \mathrm{mol} / \mathrm{L}$ CNV1014802. (Inset) The recording protocol. The currents were elicited using a range of depolarization $(-80 \mathrm{mV}$ to $+15 \mathrm{mV}$ ) from a holding potential of $-120 \mathrm{mV}$ with a stimulus frequency of $0.5 \mathrm{~Hz}$. $I-V$ relationship (B) and activation curves (C) of Nav1.7 currents with or

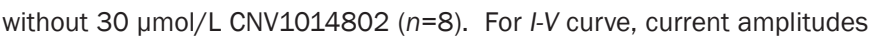
were normalized to the peak value in the absence of $30 \mu \mathrm{mol} / \mathrm{L}$ cNV1014802. 
vation of Nav1.7 channels.

\section{CNV1014802 causes a hyperpolarizing shift in the steady-state} inactivation

Next, the influences of CNV1014802 on inactivation were examined. After activation, Nav channels undergo two physically distinct inactivation processes, fast and slow inactivation $^{[25]}$. Fast inactivation is completed within a few milliseconds and can be quickly reversed after hyperpolarization. In contrast, slow inactivation develops less rapidly (time constants ranging from $\sim 100 \mathrm{~ms}$ to several minutes) and opposes high-frequency spike generation ${ }^{[25]}$. In the current study, steady-state fast inactivation of Nav1.7 channels was determined using a $500-\mathrm{ms}$ conditioning pulse from $-130 \mathrm{mV}$ to 0 $\mathrm{mV}$ in $10-\mathrm{mV}$ increments, followed by a $0-\mathrm{mV}$ test pulse. Representative traces generated in the absence or presence of 30 $\mu \mathrm{mol} / \mathrm{L}$ CNV1014802 are shown in Figure 3A. After application of the drug, the $V_{1 / 2}$ of fast inactivation was dramatically shifted by approximately $12.9 \pm 1.50 \mathrm{mV}$ in the hyperpolarizing direction (Figure 3B). The voltage-dependence of the steadystate slow inactivation was estimated using 10-s depolarization pre-pulses followed by a 20-ms hyperpolarization pulse to $-120 \mathrm{mV}$ to allow channels to recover from the fast inactivation before the test pulse to determine channel availability ${ }^{[26]}$. Providing that a recovery interval of $3 \mathrm{~s}$ at $-120 \mathrm{mV}$ is sufficient to re-prime the Nav1.7 channels after the 10-s pre-pulses (Supplementary Figure S1), a fixed 13-s pulse duration was applied during the slow inactivation study. In these conditions, $30 \mu \mathrm{mol} / \mathrm{L}$ CNV1014802 dramatically shifted the slow inactivation curve in the hyperpolarizing direction (Figure 3C). After drug administration, the $V_{1 / 2}$ of slow inactivation was changed to $-93.14 \pm 0.71 \mathrm{mV}$ from $-49.15 \pm 0.80 \mathrm{mV}$ in control conditions (Figure 3D). These data indicate that CNV1014802 mainly affects the channel inactivation.

Because CNV1014802 may inhibit the channel by stabilizing an inactivated state, the inhibition efficacies of the drug in the resting and half-inactivated states were further investigated. According to the inactivation curve of Nav1.7 shown in Figure $3 \mathrm{~B}$, holding potentials at $-120 \mathrm{mV}$ and $-70 \mathrm{mV}$ will keep the channel in the resting and half-inactivated states, respectively. The $\mathrm{IC}_{50}$ value (with $95 \%$ confidence limits) of CNV1014802 was decreased to 1.77 (1.20-2.33) $\mu \mathrm{mol} / \mathrm{L}$ from 71.66 (46.85-96.48) $\mu \mathrm{mol} / \mathrm{L}$ when half of the channels were inactivated (Figure 3E). Overall, CNV1014802 acts as a state-dependent inhibitor and preferentially binds to inactivated Nav1.7 channels.

\section{CNV1014802 shows use-dependent inhibition of Nav1.7 channels}

Use-dependent inhibition is an important property of many previously reported Nav channel inhibitors ${ }^{[27]}$. Hence, the usedependence of CNV1014802 was investigated. Cells expressing Nav1.7 were first recorded under a train of 20-ms voltage steps to $0 \mathrm{mV}$ from a $-120-\mathrm{mV}$ holding potential at frequencies of 2,10 and $20 \mathrm{~Hz}$ in the absence or presence of $10 \mu \mathrm{mol} / \mathrm{L}$ CNV1014802. No significant inhibition was observed during the 2-Hz stimulus frequency (Figure 4A). However, cumulative inhibition became evident during the higher frequency
A
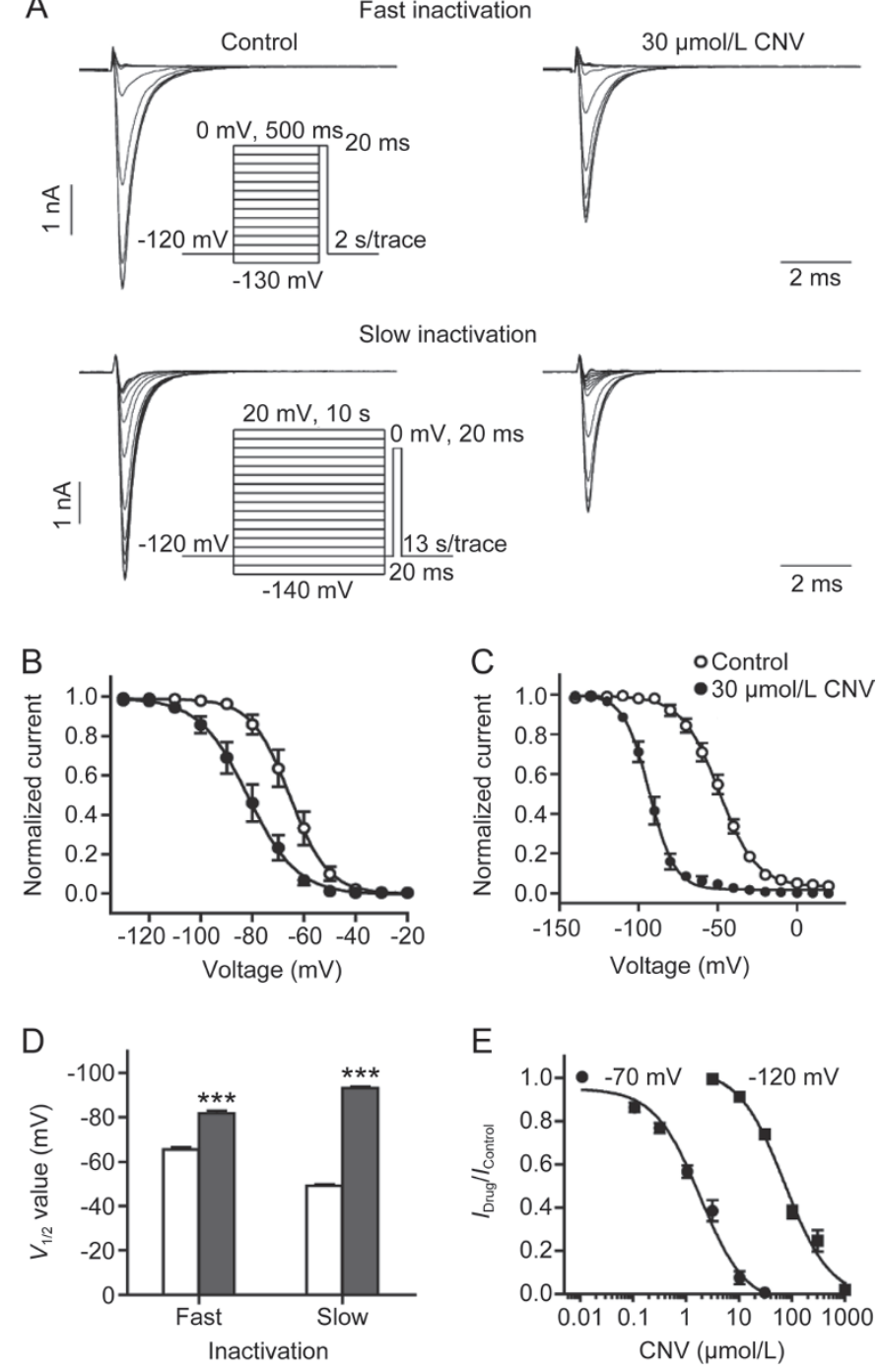

Figure 3. Characterization of the CNV1014802 modulation on Nav1.7 steady-state inactivation. (A) Representative steady-state fast (upper) and slow (lower) inactivation current traces of Nav1.7 in the absence or

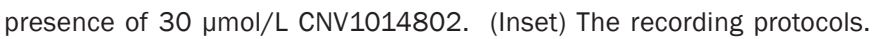
The steady-state fast inactivation was evaluated by a test step to $0 \mathrm{mV}$ after a $500 \mathrm{~ms}$ pre-pulses to a varying voltage from a holding potential of $-120 \mathrm{mV}$ with a stimulus frequency at $0.5 \mathrm{~Hz}$. The steady-state slow inactivation was examined using $10 \mathrm{~s}$ depolarization pre-pulses followed by a $20 \mathrm{~ms}$ hyperpolarization pulse to $-120 \mathrm{mV}$ to allow channels to recover from fast inactivation, and a test to $0 \mathrm{mV}$ for $20 \mathrm{~ms}$ was then used to determine the channel availability. The voltage dependence of the steady-state fast (B) and slow (C) inactivation in the absence or presence of $30 \mu \mathrm{mol} / \mathrm{L} \mathrm{CNV1014802} \mathrm{(} n=10$ for fast inactivation and $n=5$ for slow inactivation). (D) The $V_{1 / 2}$ values of the fast- and slow- inactivation summarized from panel $B$ and $C$. ${ }^{* * *} P<0.0001$, unpaired 2-tailed $t$ test. (E) Dose-response curves of CNV1014802 for Nav1.7 at holding potentials of $-70 \mathrm{mV}$ and $-120 \mathrm{mV}$. The values of inhibition by CNV1014802 at high concentrations $(\geq 300 \mu \mathrm{mol} / \mathrm{L}$ ) could not be accurately measured because of the inhibitor's poor solubility ( $n=5$ for each condition).

stimulations (Figure $4 \mathrm{~A}$ ). The inhibition was $31 \% \pm 0.9 \%$ and $55 \% \pm 1.2 \%$ for $10 \mathrm{~Hz}$ and $20 \mathrm{~Hz}$, respectively, in the presence of CNV1014802 (Figure 4B). The reductions in the Nav1.7 
A
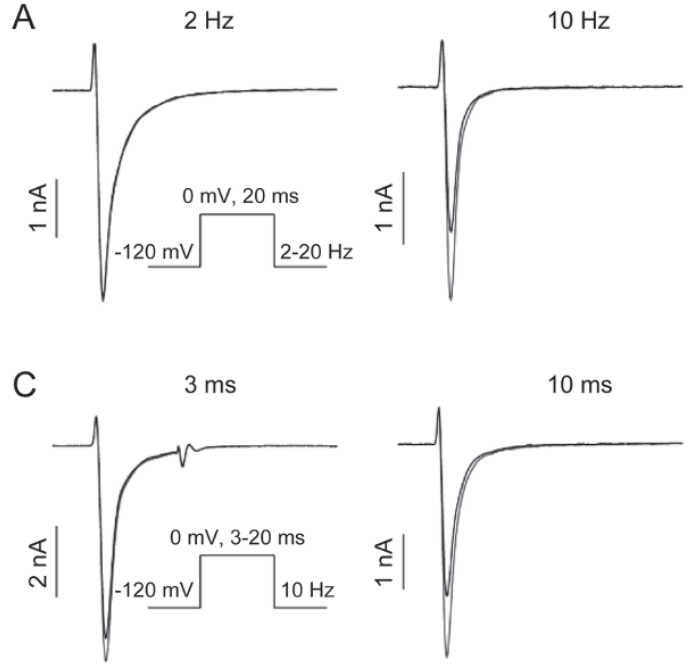
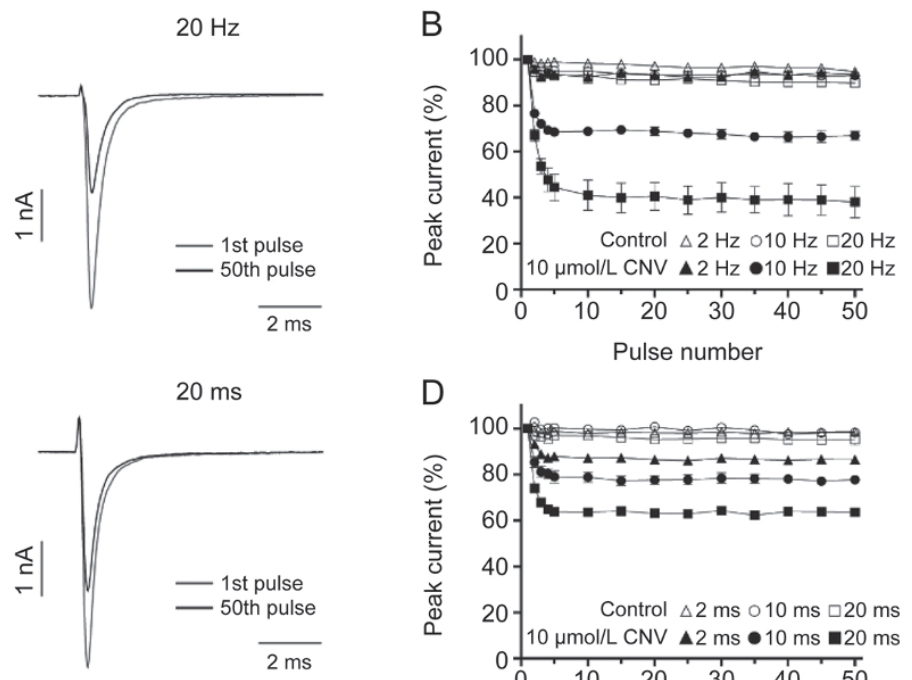

Figure 4. Use-dependent inhibition of CNV1014802 modulated by the depolarization duration. (A) Representative Nav1.7 currents recorded in the presence of $10 \mu \mathrm{mol} / \mathrm{L}$ CNV1014802 at three different stimulus frequencies. (Inset) The recording protocol. The use-dependent inhibition was evaluated at 2, 10 and $20 \mathrm{~Hz}$ with a fixed $20 \mathrm{~ms}$ test pulse to $0 \mathrm{mV}$ from a holding potential of $-120 \mathrm{mV}$. (B) Bar graph showing the relative amplitudes of the use-dependent inhibition for each frequency ( $n=5$ for each condition). Current amplitudes were normalized to the value of first trace. (C) Representative Nav1.7 currents evoked by variable test pulse durations to $0 \mathrm{mV}$ in the presence of $10 \mu \mathrm{mol} / \mathrm{L} \mathrm{CNV1014802} \mathrm{at} 10 \mathrm{~Hz}$. (Inset) The recording protocol. The use-dependent inhibition was tested at $10 \mathrm{~Hz}$ with 3,10 and $20 \mathrm{~ms}$ test pulses to $0 \mathrm{mV}$ from a holding potential of $-120 \mathrm{mV}$. (D) Bar graph showing the relative amplitudes of the use-dependent inhibition for each pulse duration ( $n=5$ for each condition). Current amplitudes were normalized to the value of first trace. To evaluate the use-dependent inhibition properties of Nav1.7 channels in the absence and presence of 10 $\mu \mathrm{mol} / \mathrm{L}$ CNV1014802, the current amplitudes were normalized to the first pulse which was presented as a filled black square for panel B and D.

current amplitudes for all stimulation frequencies in the absence of CNV1014802 were less than 10\% (Figure 4B). Thus, CNV1014802 produces a strong use-dependent inhibition of Nav1.7 channels.

In the presence of CNV1014802, repetitive stimulation might cause Nav1.7 channels to progress to an inactivated state, thereby inducing a more potent inhibition. In other words, the use-dependent inhibition of Nav1.7 channels by CNV1014802 might be modulated by channel inactivation. Because a longer pulse duration inactivates more Nav1.7 channels, we varied the stimulation duration and investigated its influence on CNV1014802 inhibition. The stimulation durations were varied from 3 to $20 \mathrm{~ms}$ at a fixed frequency of 10 $\mathrm{Hz}$. In the absence of CNV1014802, the reductions in the Nav1.7 peak currents were less than $5 \%$ in all three stimulation conditions. In contrast, after application of $10 \mu \mathrm{mol} /$ L CNV1014802, the peak currents decreased in a remarkable duration-dependent manner (Figure 4C). The current reduction ratios were $13 \% \pm 1.4 \%, 22 \% \pm 1.4 \%$ and $36 \% \pm 0.6 \%$ for 3 -, $10-$ and $20-\mathrm{ms}$ pulses, respectively (Figure $4 \mathrm{D}$ ). The frequency and duration-dependent inhibition is consistent with the notion that CNV1014802 mainly affects channel inactivation.

\section{Influences of CNV1014802 on the development of inactivation}

To better understand the interaction between CNV1014802 and the Nav1.7 channels, the time course of the block development induced by $10 \mu \mathrm{mol} / \mathrm{L}$ CNV1014802 was further evaluated. A series of pre-pulses to $0 \mathrm{mV}$ with variable durations
(10 ms to $19 \mathrm{~s}$ ) were applied to allow inhibition to develop and were followed by hyperpolarization of the cells to $-120 \mathrm{mV}$ for $20 \mathrm{~ms}$ to allow the drug-free channels to recover from fast inactivation. Finally, the cells were stepped to $0 \mathrm{mV}$ for 10 $\mathrm{ms}$, and the fraction of channels available for activation was measured. Figure 5A shows the representative current traces corresponding to various pre-pulse durations with or without

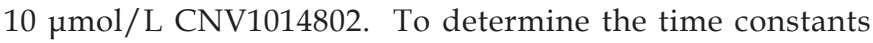
of inactivation development, the normalized amount of currents was plotted against the pre-pulse durations. Normally, in control cells, the time course represents the development of slow inactivation, which could be fitted with a one-phase exponential equation ${ }^{[28]}$. However, CNV1014802 dramatically changed the inactivation profile, which was better fitted by a two-phase exponential function. Tau 1 probably represents the time constant of fast inactivation development, whereas Tau 2 is the time constant of slow inactivation development ${ }^{[28]}$. Notably, the current amplitude decreased approximately $80 \%$ during the fast component, whereas no detectable decrease was observed for the same duration in the control (Figure 5B). In contrast to the induction of a fast inactivation component, the development of the slow inactivation was not affected by $10 \mu \mathrm{mol} / \mathrm{L}$ CNV1014802 (Table 1).

\section{CNV1014802 slows the recovery from inactivation}

We next investigated the rate of recovery of Nav1.7 channels from the inactivation inhibition by CNV1014802. A 10-s inactivating pre-pulse to $0 \mathrm{mV}$ was used to allow CNV1014802 

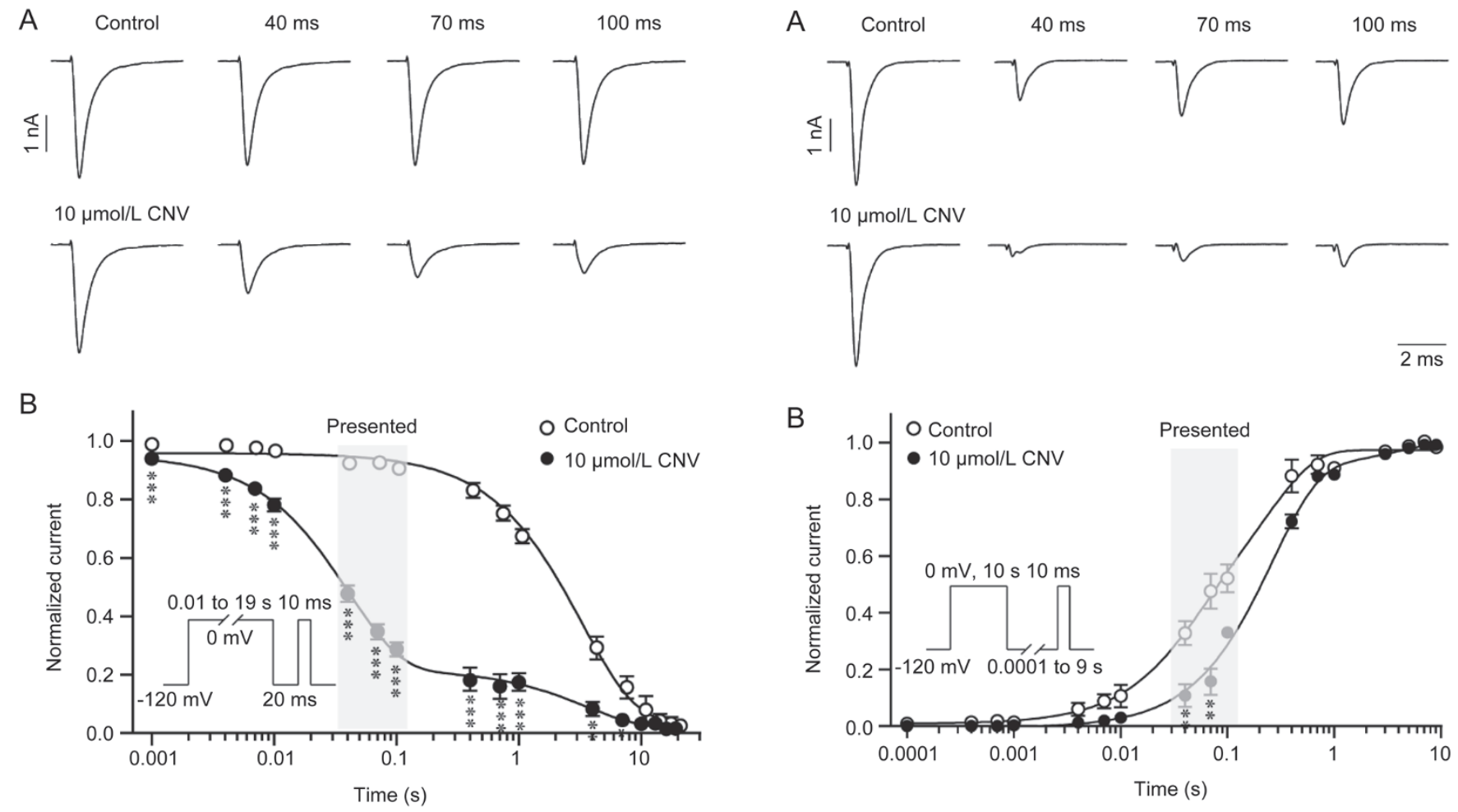

Figure 5. Effects of CNV1014802 on the development of slow inactivation in Nav1.7 channels. (A) Individual current traces from a single representative cell showing the development of Nav1.7 inactivation with or without $10 \mu \mathrm{mol} / \mathrm{L}$ CNV1014802. (B) Development of inactivation by Nav1.7 channels with or without $10 \mu \mathrm{mol} / \mathrm{L}$ CNV1014802 ( $n=6$, ${ }^{* * *} P<0.0001,{ }^{* *} P<0.001,{ }^{*} P<0.005$, paired 2 -tailed $t$ test). (Insert) The recording protocol. The entry into the inactivation state was evaluated using a two-pulse protocol consisting of a conditioning pulse of variable duration (10 ms to $19 \mathrm{~s}$ ) to $0 \mathrm{mV}$ to inactivate the channels. A $20 \mathrm{~ms}$ pulse to $-120 \mathrm{mV}$ was applied to allow channels that were not bound with drug to rapidly recover from inactivation, and a standard test pulse was used to measure the number of available channels.

inhibition to reach the steady state and was followed by a recovery interval of varying duration $(0.1 \mathrm{~ms}$ to $9 \mathrm{~s})$ at -120 $\mathrm{mV}$ before the number of available channels was measured with a standard test pulse to $0 \mathrm{mV}$ for $10 \mathrm{~ms}$. We found that $10 \mu \mathrm{mol} / \mathrm{L}$ CNV1014802 dramatically slowed the recovery of Nav1.7 channels from inactivation. In other words, the channels in the presence of CNV1014802 took longer to recover than those in the control condition (Figure 6A). The Nav1.7 channel recovery displayed a two-phase profile, regardless

Figure 6. Recovery from prolonged inactivation and CNV1014802 inhibition of Nav1.7 channels. (A) Individual current traces from a single representative cell showing the recovery of Nav1.7 channels from inactivation in the absence or presence $10 \mu \mathrm{mol} / \mathrm{L} \mathrm{CNV1014802.} \mathrm{(B)}$ Recovery of Nav1.7 channels from inactivation in the absence or presence of $10 \mu \mathrm{mol} / \mathrm{L}$ CNV1014802 ( $n=6,{ }^{* *} P<0.001$, paired 2-tailed $t$ test). (Insert) The recording protocol. Cells were held at $-120 \mathrm{mV}$, depolarized to 0 $\mathrm{mV}$ for $10 \mathrm{~s}$ and then hyperpolarized to $-120 \mathrm{mV}$ for variable increasing durations. A standard test pulse to $0 \mathrm{mV}$ for $10 \mathrm{~ms}$ was used to measure the number of available channels.

of CNV1014802 application (Figure 6B). In comparison with those in the control condition, the time constants of recovery from both fast and slow inactivation were longer after CNV1014802 application (Table 1). Together, our data suggest that both accelerating the development of inactivation and delaying the recovery from inactivation contribute to the inhibition of Nav1.7 channels by CNV1014802.

\section{CNV1014802 normalizes the functional effects of PEPD mutations}

In humans, inherited gain-of-function mutations in the Nav1.7 channel lead to severe neuropathic pain syndromes,

Table 1. Estimated time constants for the development of inhibition and recovery from inactivation block in the absence or presence of $10 \mu m o l / L$ CNV1014802.

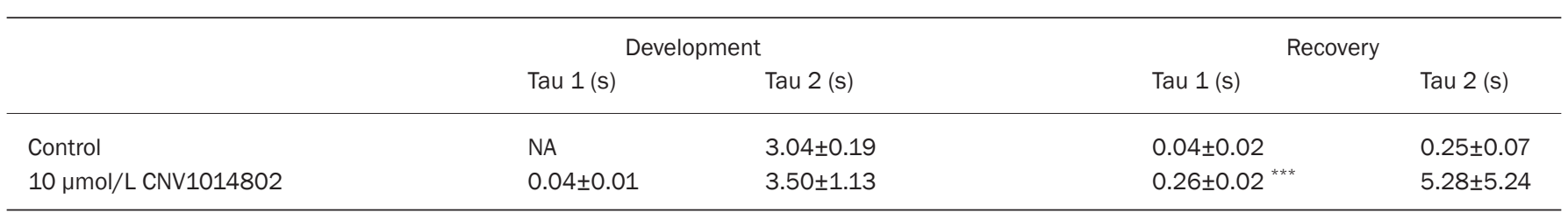

The values are presented as mean \pm SEM. NA, not available. $n=6 .{ }^{* * *} P<0.0001$, unpaired 2 -tailed $t$ test. 
such as PEPD. PEPD-linked Nav1.7 mutants mainly shift the voltage dependence of steady-state fast inactivation toward depolarization ${ }^{[6]}$. Moreover, some PEPD mutants exhibit persistent currents that may persist for more than several hundred milliseconds ${ }^{[6,29]}$. The defective fast inactivation and enhanced persistent currents increase the excitability of nociceptive neurons and thus contribute to pain $^{[6]}$. Because CNV1014802 largely shifts the inactivation of Nav1.7 channels in the hyperpolarizing direction, we speculated that the drug might be able to rescue the dampened inactivation of the PEPD mutants. To test this hypothesis, we examined the effect of $30 \mu \mathrm{mol} / \mathrm{L}$ CNV1014802 on four PEPD mutants, including F1462V, T1464I, M1627K and A1632E, using electrophysiological recording. In agreement with previous findings, these mutants, compared to the wild-type channel, produced a dramatic depolarizing shift in the fast inactivation curve ${ }^{[6,30,31]}$ (Figure 7, Table 2). Intriguingly, after $30 \mu \mathrm{mol} / \mathrm{L}$ CNV1014802 application, the dampened fast inactivation curves of all of these mutants were moved in the hyperpolarizing direction and nearly overlapped with that of the wild-type channel (Figure 7, Table 2). In addition, $30 \mu \mathrm{mol} / \mathrm{L}$ CNV1014802 did not produce visible effects on the activation of all tested mutants, consistent with the observation in the wild-type channels (Figure 7, Table 2). Notably, the persistent currents produced by T1464I and A1632E were also completely suppressed by $30 \mu \mathrm{mol} / \mathrm{L}$ CNV1014802 (Figure 7E, 7F). Collectively, our results indicate that CNV1014802 reverses the right-shifted inactivation and blocks the persistent currents caused by PEPD mutants.

\section{Discussion}

In the present study, we provide evidence that inhibition of Nav1.7 channels by CNV1014802 is attributed to enhance channel inactivation. The following novel findings support this statement: (1) CNV1014802 dramatically shifts the channel inactivation curve in the hyperpolarizing direction but
A
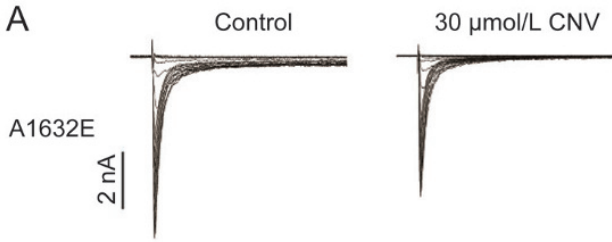

B
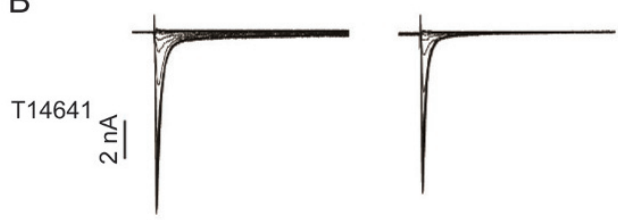

C
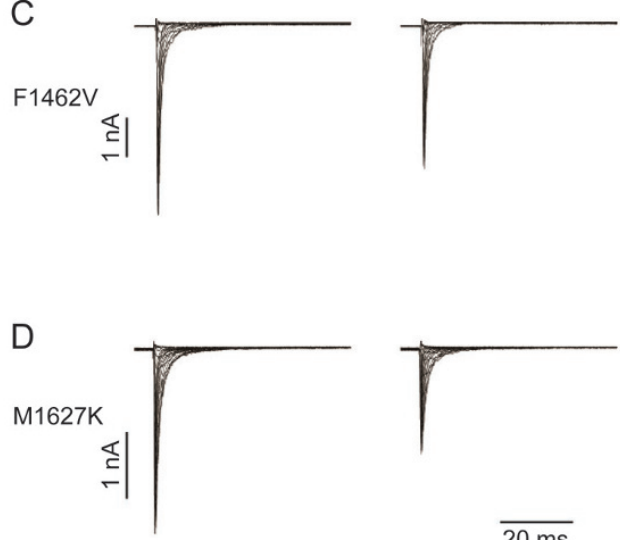
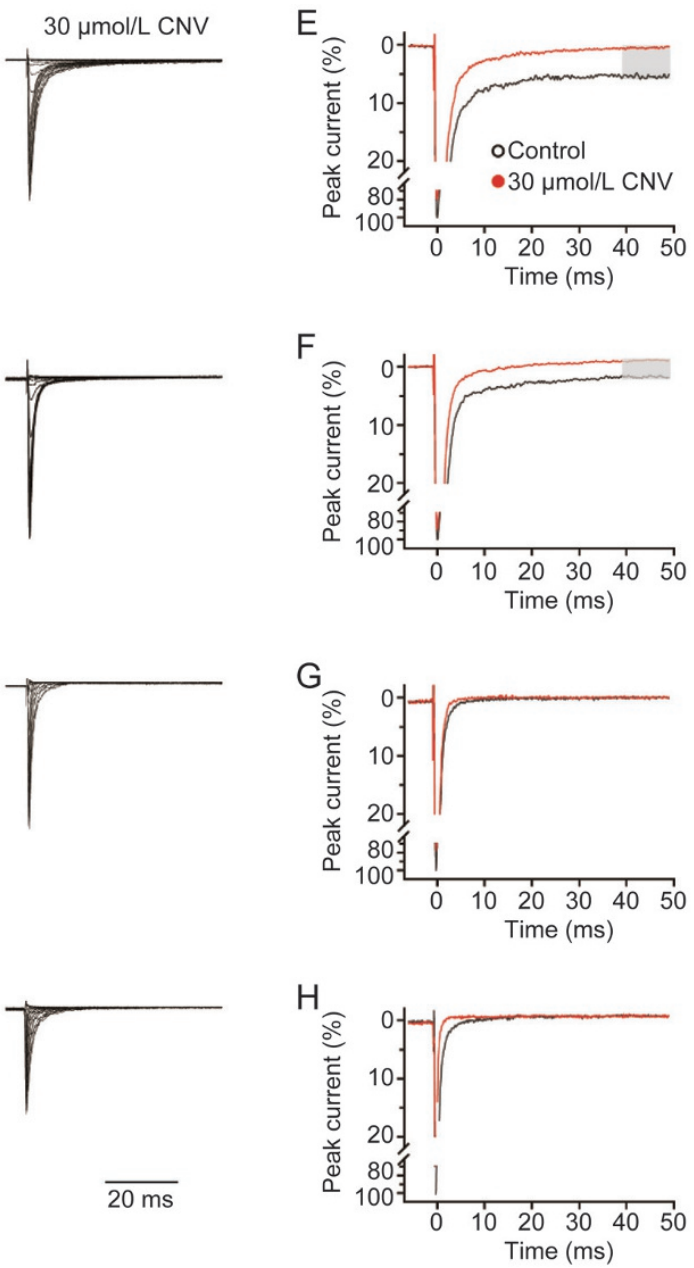
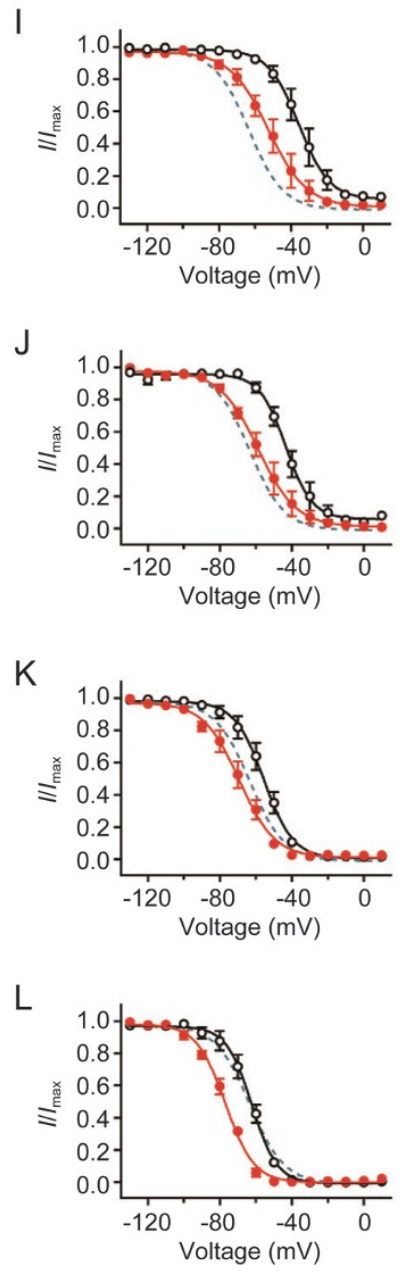

Figure 7. CNV1014802 effects on PEPD mutant channels. Representative activation currents of A1632E (A), T1464I (B), F1462V (C) and M1627K (D)

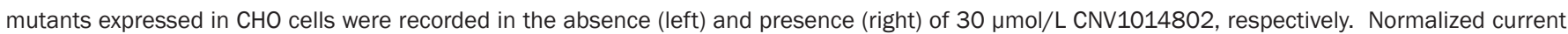
traces for A1632E (E), T1464I (F), F1462V (G) and M1627K (H) with (red line) and without (black line) 30 umol/L CNV1014802 evoked by a depolarization pulse to $0 \mathrm{mV}$. The voltage dependence of steady-state fast inactivation of A1632E (I), T1464I (J), F1462V (K) and M1627K (L) in the absence (black line) and presence (red line) of $30 \mu \mathrm{mol} / \mathrm{L}$ CNV1014802. The dashed blue line indicates the steady-state fast inactivation curve of wild type Nav1.7 channels. The recording protocols were identical to those for the wild type. The detailed information including group size was presented in Table 2. 
Table 2. Effects of $30 \mu \mathrm{mol} / \mathrm{L}$ CNV1014802 on the steady-state activation and fast inactivation parameters for PEPD-causing mutants.

\begin{tabular}{|c|c|c|c|c|c|c|}
\hline \multirow[t]{2}{*}{ Mutants } & \multicolumn{3}{|l|}{ Activation } & \multicolumn{3}{|l|}{ Inactivation } \\
\hline & Control (mV) & CNV1014802 (mV) & $n$ & Control (mV) & CNV1014802 (mV) & $n$ \\
\hline WT & $-11.62 \pm 1.15$ & $-9.94 \pm 1.11$ & 4 & $-63.34 \pm 1.52$ & $-70.16 \pm 1.29^{* *}$ & 6 \\
\hline F1462V & $-25.83 \pm 1.33$ & $-27.22 \pm 1.19$ & 4 & $-55.51 \pm 0.90$ & $-69.24 \pm 1.06^{* * *}$ & 4 \\
\hline T1464I & $-13.18 \pm 1.44$ & $-16.77 \pm 1.98$ & 7 & $-43.32 \pm 1.68$ & $-59.44 \pm 1.38^{* * *}$ & 7 \\
\hline M1627K & $-33.51 \pm 0.59$ & $-35.82 \pm 1.14$ & 5 & $-62.24 \pm 0.71$ & $-76.98 \pm 0.55^{* * *}$ & 3 \\
\hline A1632E & $-17.32 \pm 1.48$ & $-15.69 \pm 1.35$ & 4 & $-35.61 \pm 1.35$ & $-52.65 \pm 1.56^{* * *}$ & 6 \\
\hline
\end{tabular}

All values were recorded from $\mathrm{CHO}$ cells expressing Nav1.7 mutants and wild type (WT) in the absence and presence of $30 \mu \mathrm{mol} / \mathrm{L}$ CNV1014802. The values are presented as mean \pm SEM. ${ }^{* * *} P<0.001,{ }^{* * *} P<0.0001$, unpaired 2 -tailed $t$ test.

does not affect activation. (2) The use-dependent inhibition is dramatically modulated by channel inactivation in addition to stimulus frequency. (3) CNV1014802 accelerates the development of inactivation and delays the recovery from inactivation. Thus, stabilizing Nav1.7 channels in the nonconductive inactivated state may benefit the analgesic effects of CNV1014802.

The analgesic efficacy and tolerability of CNV1014802 have been investigated in a randomized, double-blind, placebocontrolled phase 2 trial for patients with $\mathrm{LSR}^{[23]}$. The clinical outcomes demonstrated that patients who orally administered CNV1014802 at a dose of $350 \mathrm{mg}$ twice per day showed a remarkable reduction in pain compared to those given placebo ${ }^{[23]}$. No severe or serious adverse events related to the drug were observed ${ }^{[23]}$. These positive results were also reported in a recently completed phase $2 \mathrm{a}$ trial for patients with trigeminal neuralgia ${ }^{[32]}$, which further indicated that CNV1014802 is well tolerated. The safety of a drug is often correlated with its selectivity. There are two reports on the selectivity of CNV1014802 $2^{[15,22]}$. One report by Zakrzewska and her colleagues stated that CNV1014802 shows selectivity for the Nav1.7 channels over the other Nav isoforms ${ }^{[22]}$. In contrast, Deuis and her colleagues claimed that CNV1014802 is a relatively non-selective Nav inhibitor using a Fluorometric Imaging Plate Reader (FLIPR) membrane potential assay ${ }^{[15]}$. Due to the limited data reported and the relatively low temporal resolution of FLIPR, we speculated that the discrimination between the two studies might be ascribed to the applied methods and cell lines ${ }^{[33]}$. The less than ideal selectivity and low affinity of CNV1014802 may limit its application and cause side effects in clinical use, which should be more closely studied in clinical trials. Nevertheless, CNV1014802 undoubtedly inhibits Nav1.7 channels, and this inhibitory effect contributes to its clinical efficacy for pain relief.

The Nav1.7 channel has been confirmed to play a central role in human pain signaling ${ }^{[4]}$. The discovery of pain-causing mutations that disrupt activation or inactivation states further support this notion ${ }^{[5-8]}$. Reducing activation may be beneficial in pain relief ${ }^{[34]}$. S241T, a gain-of-function mutation identified in a family with IEM, primarily enhances channel activation ${ }^{[35]}$. Carbamazepine, a non-selective Nav channel blocker, normalizes the enhanced activation of S241T but does not affect inactivation $^{[35]}$. A clinical study demonstrated that carbamazepine attenuates neuropathic pain in patients carrying the S241T mutation $^{[36]}$. However, the lack of efficacy of ProTx-II and SVmab1, which only affect channel activation, casts doubt on the feasibility of this approach ${ }^{[15-17]}$.

Compared with reducing activation, enhancing inactivation to relieve pain might be more feasible. Sodium channels typically undergo inactivated states during pain attacks and thus provide available binding sites for inhibitors ${ }^{[12,37]}$. Usedependent inhibition occurs when neurons fire frequently and causes the accumulation of functionally blocked channels ${ }^{[27]}$. Furthermore, the use-dependent inhibition of some of the Nav channel blockers is dependent on channel inactivation $^{[27]}$. Thus, the enhanced ability of a sodium channel blocker to discriminate between the resting and inactivated channels may provide a better safety profile and tolerability during systematic administration ${ }^{[27,28]}$. Moreover, slow closedstate inactivation but not activation allows Nav1.7 channels to boost subthreshold stimuli ${ }^{[4,13]}$. Thus, enhancing channel inactivation may be much more efficient at depressing neuron excitability. In addition, modulating inactivation might be useful in the treatment of pain syndromes caused by disrupted activation $^{[38]}$. For example, the mutation R1279P causes both gain-of-function depolarized inactivation and loss-of-function depolarized activation ${ }^{[38]}$. However, this mutation in humans causes distal limb pain, which suggests that the gain-offunction depolarized inactivation is dominant and overrides the influence of the impaired activation ${ }^{[38]}$. Overall, modulating channel inactivation is a feasible approach for pain management, which is consistent with the positive data in clinical trials and the mechanism of CNV1014802 revealed in the current study.

Previous studies have investigated how a Nav1.7 channel inhibitor interacts with the inactivated conformations. Lidocaine, a classical local anesthetic, has been reported to decrease the transition of Nav1.7 channels to the slow inactivation state $^{[26]}$. The time courses of recovery from inactivation with or without lidocaine overlap with one another ${ }^{[26]}$. This feature was not observed in CNV1014802. Previously, lacosamide was reported to primarily target channels in the slow inactivation state ${ }^{[28]}$. Rather than affecting the slow inactivation state, lacosamide is currently thought to induce its inhibitory effect by its slow binding to fast-inactivated states ${ }^{[39]}$. Nevertheless, the mechanism underlying lacosamide inhibition is obviously different from that of CNV1014802, which left-shifts both fast and slow inactivation and causes a fast inhibition onset 
(Figure 3 and 5). Carbamazepine, another reported sodium channel inhibitor, has been reported to interact with Nav1.7 in its inactivated state ${ }^{[28]}$. Although carbamazepine and CNV1014802 change the development of slow inactivation in a similar manner, the compounds have different effects on the recovery from inactivation ${ }^{[28]}$. The recovery from inactivation is prominently delayed by $10 \mu \mathrm{mol} / \mathrm{L} \mathrm{CNV1014802} \mathrm{(Figure} \mathrm{6).}$ In contrast, carbamazepine does not change the recovery time course of Nav1.7 inactivation ${ }^{[28]}$. Trapping Nav1.7 channels in a nonconductive state was also suggested for PF-05089771 and its analogue PF-04856264 ${ }^{[19]}$. In contrast to CNV1014802, which takes 30-60 s to reach steady-state inhibition of Nav1.7 channels, PF-05089771 takes approximately $17 \mathrm{~min}$ to reach a steady state of inhibition ${ }^{[19]}$. Moreover, the development of inhibition in the presence of PF-05089771 is well fitted by a one-phase exponential equation ${ }^{[19]}$, whereas CNV1014802 causes a two-phase profile (Figure 5). Considering the stateand use-dependent properties of CNV1014802, it could act as an open channel blocker similar to local anesthetics ${ }^{[40]}$. However, PF-04856264, a selective Nav1.7 channel inhibitor that interacts with the S1-S4 voltage sensor of domain IV, also inhibits the channel in state- and use-dependent manners ${ }^{[41,42]}$. Thus, a following binding site study will be useful to test this possibility. Overall, CNV1014802 interacts with Nav1.7 channels in a manner distinct manner from previously reported inhibitors that affect channel inactivation.

Classically, PEPD-causing mutations shift the fast inactivation in the depolarizing direction, thus allowing more channels to be available at a defined membrane potential ${ }^{[6]}$. Other changes may include persistent currents, enhanced resurgent currents, slower current decay and increased recovery from inactivation. Resurgent currents are proposed to be mediated by an endogenous blocking particle, primarily assumed to be the C-terminal tail of the auxiliary Nav $\beta 4$ subunit ${ }^{[43]}$. Moreover, drug-mediated inhibition of resurgent current inhibition is well correlated with inhibition of persistent currents ${ }^{[29]}$. This observation indicates that a compound that inhibits a persistent current may block a resurgent current as well ${ }^{[29]}$. Some PEPD patients derive benefits from carbamazepine because the drug rescues the impaired fast inactivation and blocks the persistent currents ${ }^{[6,30]}$. However, the responses to carbamazepine and other available sodium channel blockers are inconsistent, and some of these patients even become refractory to these therapeutics ${ }^{[44]}$. In the present study, we selected 4 PEPD mutants and examined how CNV1014802 affected them. The mutations F1462V and T1464I are located in the linker region between domains III and IV. Furthermore, F1462V occurs within the completely conserved IFM fast inactivation motif among all Nav isoforms ${ }^{[6]}$. The mutation M1627K is located within the domain IV S4-S5 linker and may form part of the inactivation gate ${ }^{[30]}$. In contrast to these 3 mutations causing PEPD syndrome in patients, the A1632E mutation produces mixed symptoms of IEM and PEPD. The A1632 residue within the linker between the S4-S5 linker of domain IV is highly conserved among all sodium channels except Nav1.9 ${ }^{[31]}$. We found that CNV1014802 is capable of restoring the disrupted function caused by these PEPD mutations, including the dampened inactivation or the persistent currents (Figure 7). Thus, CNV1014802 may offer an alternative therapeutic option for PEPD patients. These data provide a preliminary experimental basis for the expansion of the clinical application of CNV1014802.

In conclusion, we systematically investigated CNV1014802, and we found that enhancing Nav1.7 channel inactivation contributes to its pharmacological inhibition. Given its tolerance and efficacy in chronic pain treatment, our data suggest that enhancing inactivation could be considered an appropriate approach for drug development against Nav1.7 channels. The functions of normalizing the right-shifted inactivation and blocking the persistent currents of the gain-of-function mutations make CNV1014802 an alternative and available candidate drug for PEPD therapy.

\section{Acknowledgements}

We thank Dr Norbert KLUGBAUER (Albert-Ludwigs-Universität Freiburg, Freiburg, Germany) for the gift of hNav1.7 cDNA. This work was supported in part by a postdoctoral fellowship award from China's Post-Doctoral Science Fund (2016M591728) (to Yue-ming ZHENG), the National Key Scientific Instrument \& Equipment Development Program of China (2012YQ03026010) and the National Natural Science Foundation of China (61327014 and 61175103) (to Zhao-bing GAO).

\section{Author contribution}

Yue-ming ZHENG and Zhao-bing GAO designed and conceived the experiments; Yue-ming ZHENG, Wan-fu WANG and Yan-fen LI performed the experiments; all authors analyzed the data; Yue-ming ZHENG, Yong YU and Zhao-bing GAO wrote the paper.

\section{Supplementary information}

Supplementary information is available on the website of Acta Pharmacologica Sinica

\section{References}

1 Yu FH, Catterall WA. Overview of the voltage-gated sodium channel family. Genome Biol 2003; 4: 207.

2 Klugbauer N, Lacinova L, Flockerzi V, Hofmann F. Structure and functional expression of a new member of the tetrodotoxin-sensitive voltage-activated sodium channel family from human neuroendocrine cells. EMBO J 1995; 14: 1084-90.

3 Djouhri L, Newton R, Levinson SR, Berry CM, Carruthers B, Lawson SN. Sensory and electrophysiological properties of guinea-pig sensory neurones expressing Nav 1.7 (PN1) $\mathrm{Na}^{+}$channel alpha subunit protein. J Physiol 2003; 546: 565-76.

4 Dib-Hajj SD, Yang Y, Black JA, Waxman SG. The $\mathrm{Na}(\mathrm{V}) 1.7$ sodium channel: from molecule to man. Nat Rev Neurosci 2013; 14: 49-62.

5 Yang Y, Wang Y, Li S, Xu Z, Li H, Ma L, et al. Mutations in SCN9A, encoding a sodium channel alpha subunit, in patients with primary erythermalgia. J Med Genet 2004; 41: 171-4.

6 Fertleman CR, Baker MD, Parker KA, Moffatt S, Elmslie FV, Abrahamsen B, et al. SCN9A mutations in paroxysmal extreme pain disorder: allelic variants underlie distinct channel defects and phenotypes. Neuron 2006; 52: 767-74.

7 Faber CG, Hoeijmakers JG, Ahn HS, Cheng X, Han C, Choi JS, et 
al. Gain of function Nav1.7 mutations in idiopathic small fiber neuropathy. Ann Neurol 2012; 71: 26-39.

8 Cox JJ, Reimann F, Nicholas AK, Thornton G, Roberts E, Springell K, et al. An SCN9A channelopathy causes congenital inability to experience pain. Nature 2006; 444: 894-8.

9 Kurban M, Wajid M, Shimomura Y, Christiano AM. A nonsense mutation in the SCN9A gene in congenital insensitivity to pain. Dermatology 2010; 221: 179-83.

10 Bagal SK, Chapman ML, Marron BE, Prime R, Storer RI, Swain NA. Recent progress in sodium channel modulators for pain. Bioorg Med Chem Lett 2014; 24: 3690-9.

11 Emery EC, Luiz AP, Wood JN. Nav1.7 and other voltage-gated sodium channels as drug targets for pain relief. Expert Opin Ther Targets 2016; 20: 975-83.

12 Vetter I, Deuis JR, Mueller A, Israel MR, Starobova H, Zhang A, et al. NaV1.7 as a pain target - from gene to pharmacology. Pharmacol Ther 2017; 172: 73-100.

13 Cummins TR, Howe JR, Waxman SG. Slow closed-state inactivation: a novel mechanism underlying ramp currents in cells expressing the hNE/PN1 sodium channel. J Neurosci 1998; 18: 9607-19.

14 Lee JH, Park CK, Chen G, Han Q, Xie RG, Liu T, et al. A monoclonal antibody that targets a NaV1.7 channel voltage sensor for pain and itch relief. Cell 2014; 157: 1393-404.

15 Deuis JR, Wingerd JS, Winter Z, Durek T, Dekan Z, Sousa SR, et al. Analgesic effects of GpTx-1, PF-04856264 and CNV1014802 in a mouse model of NaV1.7-mediated pain. Toxins (Basel) 2016; 8. pii: E78.

16 Liu D, Tseng M, Epstein LF, Green L, Chan B, Soriano B, et al. Evaluation of recombinant monoclonal antibody SVmab1 binding to NaV1.7 target sequences and block of human NaV1.7 currents. F1000Res 2016; 5: 2764.

17 Schmalhofer WA, Calhoun J, Burrows R, Bailey T, Kohler MG, Weinglass $A B$, et al. ProTx-II, a selective inhibitor of NaV1.7 sodium channels, blocks action potential propagation in nociceptors. Mol Pharmacol 2008; 74: 1476-84.

18 Ahuja S, Mukund S, Deng L, Khakh K, Chang E, Ho H, et al. Structural basis of Nav1.7 inhibition by an isoform-selective small-molecule antagonist. Science 2015; 350: aac5464.

19 Theile JW, Fuller MD, Chapman ML. The selective Nav1.7 inhibitor, PF-05089771, interacts equivalently with fast and slow inactivated Nav1.7 channels. Mol Pharmacol 2016; 90: 540-8.

20 Cao L, McDonnell A, Nitzsche A, Alexandrou A, Saintot PP, Loucif AJ, et al. Pharmacological reversal of a pain phenotype in iPSC-derived sensory neurons and patients with inherited erythromelalgia. Sci Transl Med 2016; 8: 335ra56.

21 Waxman SG, Merkies IS, Gerrits MM, Dib-Hajj SD, Lauria G, Cox JJ, et al. Sodium channel genes in pain-related disorders: phenotypegenotype associations and recommendations for clinical use. Lancet Neurol 2014; 13: 1152-60.

22 Zakrzewska JM, Palmer J, Ettlin DA, Obermann M, Giblin GM, Morisset $\checkmark$, et al. Novel design for a phase lla placebo-controlled, double-blind randomized withdrawal study to evaluate the safety and efficacy of CNV1014802 in patients with trigeminal neuralgia. Trials 2013; 14: 402.

23 Versavel M. Efficacy and safety of the novel sodium channel blocker CNV1014802 in trigeminal neuralgia and lumbosacral radiculopathy. J Pain Relief 2015. DOI: 10.4172/2167-0846.S1.002.

24 Curtis MJ, Bond RA, Spina D, Ahluwalia A, Alexander SP, Giembycz $\mathrm{MA}$, et al. Experimental design and analysis and their reporting: new guidance for publication in BJP. Br J Pharmacol 2015; 172: 3461-71.

25 Karoly R, Lenkey N, Juhasz AO, Vizi ES, Mike A. Fast- or slowinactivated state preference of $\mathrm{Na}^{+}$channel inhibitors: a simulation and experimental study. PLoS Comput Biol 2010; 6: e1000818.

26 Sheets PL, Jarecki BW, Cummins TR. Lidocaine reduces the transition to slow inactivation in $\mathrm{Na}(\mathrm{v}) 1.7$ voltage-gated sodium channels. $\mathrm{Br} \mathrm{J}$ Pharmacol 2011; 164: 719-30.

27 England S, de Groot MJ. Subtype-selective targeting of voltage-gated sodium channels. Br J Pharmacol 2009; 158: 1413-25.

28 Sheets PL, Heers C, Stoehr T, Cummins TR. Differential block of sensory neuronal voltage-gated sodium channels by lacosamide [(2R)2-(acetylamino)-N-benzyl-3-methoxypropanamide], lidocaine, and carbamazepine. J Pharmacol Exp Ther 2008; 326: 89-99.

29 Theile JW, Cummins TR. Inhibition of Navbeta4 peptide-mediated resurgent sodium currents in Nav1.7 channels by carbamazepine, riluzole, and anandamide. Mol Pharmacol 2011; 80: 724-34.

30 Dib-Hajj SD, Estacion M, Jarecki BW, Tyrrell L, Fischer TZ, Lawden M, et al. Paroxysmal extreme pain disorder M1627K mutation in human Nav1.7 renders DRG neurons hyperexcitable. Mol Pain 2008; 4: 37.

31 Estacion M, Dib-Hajj SD, Benke PJ, Te Morsche RH, Eastman EM, Macala $\amalg$, et al. NaV1.7 gain-of-function mutations as a continuum: A1632E displays physiological changes associated with erythromelalgia and paroxysmal extreme pain disorder mutations and produces symptoms of both disorders. J Neurosci 2008; 28: 11079-88.

32 Zakrzewska JM, Palmer J, Morisset V, Giblin GM, Obermann M, Ettlin DA, et al. Safety and efficacy of a Nav1.7 selective sodium channel blocker in patients with trigeminal neuralgia: a double-blind, placebocontrolled, randomised withdrawal phase 2a trial. Lancet Neurol 2017; 16: 291-300.

33 Yu HB, Li M, Wang WP, Wang XL. High throughput screening technologies for ion channels. Acta Pharmacol Sin 2016; 37: 34-43.

34 Fischer TZ, Gilmore ES, Estacion M, Eastman E, Taylor S, Melanson M, et al. A novel Nav1.7 mutation producing carbamazepine-responsive erythromelalgia. Ann Neurol 2009; 65: 733-41.

35 Yang Y, Dib-Hajj SD, Zhang J, Zhang Y, Tyrrell L, Estacion M, et al. Structural modelling and mutant cycle analysis predict pharmacoresponsiveness of a $\mathrm{Na}(\mathrm{V}) 1.7$ mutant channel. Nat Commun 2012; 3: 1186.

36 Geha P, Yang Y, Estacion M, Schulman BR, Tokuno H, Apkarian $A V$, et al. Pharmacotherapy for pain in a family with inherited erythromelalgia guided by genomic analysis and functional profiling. JAMA Neurol 2016; 73: 659-67.

37 Dick IE, Brochu RM, Purohit Y, Kaczorowski GJ, Martin WJ, Priest BT. Sodium channel blockade may contribute to the analgesic efficacy of antidepressants. J Pain 2007; 8: 315-24.

38 Huang J, Yang Y, Dib-Hajj SD, van Es M, Zhao P, Salomon J, et al. Depolarized inactivation overcomes impaired activation to produce DRG neuron hyperexcitability in a Nav1.7 mutation in a patient with distal limb pain. J Neurosci 2014; 34: 12328-40.

39 Jo S, Bean BP. Lacosamide inhibition of Nav1.7 voltage-gated sodium channels: slow binding to fast-inactivated states. Mol Pharmacol 2017; 91: 277-86.

40 Scholz A. Mechanisms of (local) anaesthetics on voltage-gated sodium and other ion channels. Br J Anaesth 2002; 89: 52-61.

41 McCormack K, Santos S, Chapman ML, Krafte DS, Marron BE, West $\mathrm{CW}$, et al. Voltage sensor interaction site for selective small molecule inhibitors of voltage-gated sodium channels. Proc Natl Acad Sci U S A 2013; 110: E2724-32.

42 Zhang $\mathrm{H}$, Reichert E, Cohen AE. Optical electrophysiology for probing function and pharmacology of voltage-gated ion channels. Elife 2016; 5. pii: e15202.

43 Theile JW, Cummins TR. Recent developments regarding voltagegated sodium channel blockers for the treatment of inherited and acquired neuropathic pain syndromes. Front Pharmacol 2011; 2: 54.

44 Choi JS, Boralevi F, Brissaud O, Sanchez-Martin J, Te Morsche RH, DibHajj SD, et al. Paroxysmal extreme pain disorder: a molecular lesion of peripheral neurons. Nat Rev Neurol 2011; 7: 51-5. 